\title{
A Case Report of Full-Mouth Rehabilitation of Amelogenesis Imperfecta with Improved Oral Health Impact Profile
}

\author{
Basnet BB* , Parajuli PK, Singh RK, Suwal P \\ Department of Prosthodontics, B. P. Koirala Institute of Health Sciences, Dharan, Nepal \\ *Corresponding author: bishal_bpkihs@yahoo.com
}

Received September 22, 2014; Revised October 20, 2014; Accepted October 26, 2014

\begin{abstract}
Amelogenesis imperfecta is a hereditary disorder of tooth formation that affects the enamel layer of teeth resulting in poor dental esthetics, chipped off tooth structure with dentinal hypersensitivity. Treatment of such cases demands full-mouth rehabilitation with tooth colored ceramic crowns after endodontic treatment of the symptomatic teeth. This case describes the full-mouth rehabilitation of a 19-year-old male with significantly improved oral health impact profile. The hereditary disorder of tooth formation, if treated meticulously after proper clinical and radiographic diagnosis results in better oral health impact profile.
\end{abstract}

Keywords: Amelogenesis imperfecta, full-mouth rehabilitation, oral health impact profile

Cite This Article: Basnet BB, Parajuli PK, Singh RK, and Suwal P, "A Case Report of Full-Mouth Rehabilitation of Amelogenesis Imperfecta with Improved Oral Health Impact Profile.” International Journal of Dental Sciences and Research, vol. 2, no. 6 (2014): 133-136. doi: 10.12691/ijdsr-2-6-3.

\section{Introduction}

Amelogenesis imperfecta, also known as herediatary opalescent teeth, represents a group of herediatary defects of enamel unassociated with any other generalized defects. It is entirely ectodermal in origin and mutations in AMLEX (amelogenin), ENAM (enamelin), KLK4 (Kallikrein 4), MMP-20(enamelysin) are thought to be responsible for this defect. [1,2] Amelogenesis imperfecta can be classified into four types, but it was later revised by Witkop and given various subclasses. [3] The clinical features may range from poor esthetics, brown pigmentation, decreased vertical dimension to even painful sensitivity to teeth. The teeth are vulnerable to pulpal inflammation. Enamel maybe entirely absent in radiograph, if present it will be very thin layer usually at the cusp tips and interproximal surfaces. Sometimes, radiograph shows indistinct enamel with equal radiolucency to dentin [1].

The prevalence of AI is 1 in 14 people to 1.14 per 1000 depending on type, [2] 1:718 to 1:14000 in western countries [4] and in our part of the world very rare; $0.27 \%$ in Indian population [5].

The restoration of AI involves meticulous treatment planning and execution of high-standard of treatment protocol. There are often two schools of thoughts to deal with such cases. Twin table concept was developed by Dr. Sumiya Hobo which develops the anterior guidance to create a pre-determined, harmonious disclusion with the condylar path. The technique utilizes 2 different customized incisal guide tables. The first one is incisal table without disclusion and the second is termed incisal guidance with disclusion created by placing $3 \mathrm{~mm}$ plastic separators behind the condylar elements. [6,7] Another philosophy advocates a static co-ordinated contact of aximum number of teeth when mandible is in centric relation. It harmonizes the anterior guidance for best possible esthetics, function and comfort. A suitable occlusal plane is selected, according to which lower posteriors are restored and finally upper posteriors are restored in harmony with the anterior guidance and condylar guidance [8].

\section{Case Description}

This case report describes prosthetic rehabilitation of a 19-year-old male who reported to Department of Prosthodontics, B. P. Koirala Institute of Health Sciences, Dharan, Nepal with chief complaints of ugly appearance of teeth and sensitivity to some of his teeth. His family history revealed similar problem in his father and his paternal aunt (Figure 1). He was asked to fill a questionnaire regarding his problem according to Oral Health Impact Profile-14. This was developed with the aim of providing comprehensive measure of self-reported dysfunction, discomfort and disability attributed to oral conditions $[9,10]$.

The patient had poorer oral health impact (Figure 2) prior to the treatment. The case was diagnosed to be hypocalcified type of AI. His orthopantograph (Figure 4) showed endodontic therapy of his anterior teeth, which he had undergone for sensitivity in a local clinic. The treatment protocol was formulated according to the 
Pankey Mann and Schuyler philosophy. Some of his teeth were judged to be candidates for endodontic treatment (Figure 5, Figure 6). The face-bow record was obtained and transferred to a semi-adjustable articulator (Figure 7, Figure 8), anterior teeth are restored to please the functions and esthetics. Anterior guidance was determined. Later on posterior teeth are prepared and restoration was done with metal-ceramic single unit crowns. The patient was again asked to fill the questionnaire for the prostheses. It was seen great improvement of his oral health (Table 1).

Table 1. the analysis of scores of OHIP-14

\begin{tabular}{|c|c|c|}
\hline Dimension & Pre-treatment & Post-treatment \\
\hline Functional limitation & 0 & 0 \\
\hline Physical pain & 2.34 & 0 \\
\hline Psychological discomfort & 1.11 & 0.45 \\
\hline Physical disability & 0 & 0 \\
\hline Psychological disability & 1.6 & 0 \\
\hline Social disability & 0.76 & 0 \\
\hline Handicap & 1.18 & 0 \\
\hline
\end{tabular}

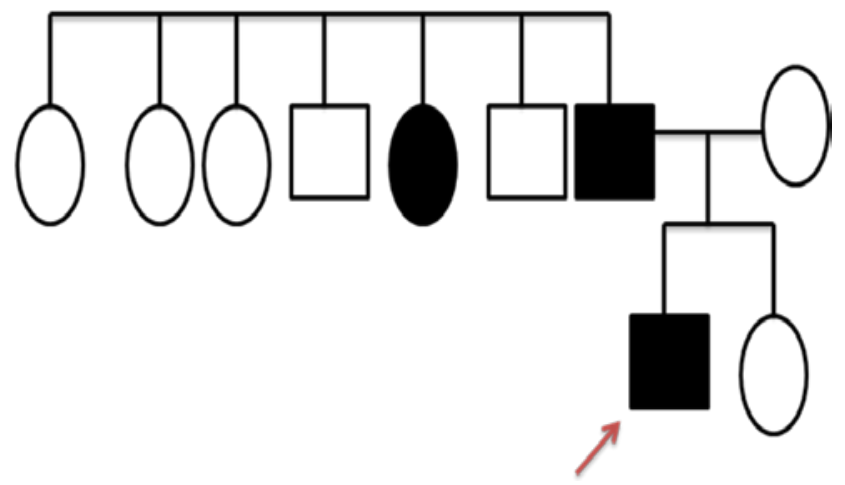

Figure 1. The pedigree analysis of the patient(arrow)

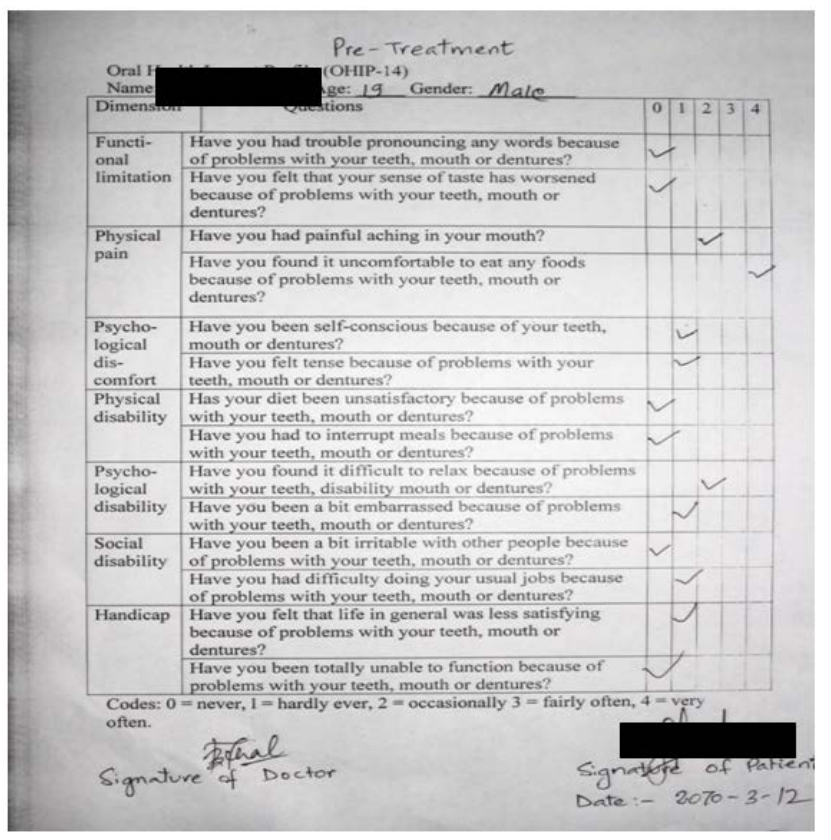

Figure 2. Pre-treatment OHIP-14 filled by the patient

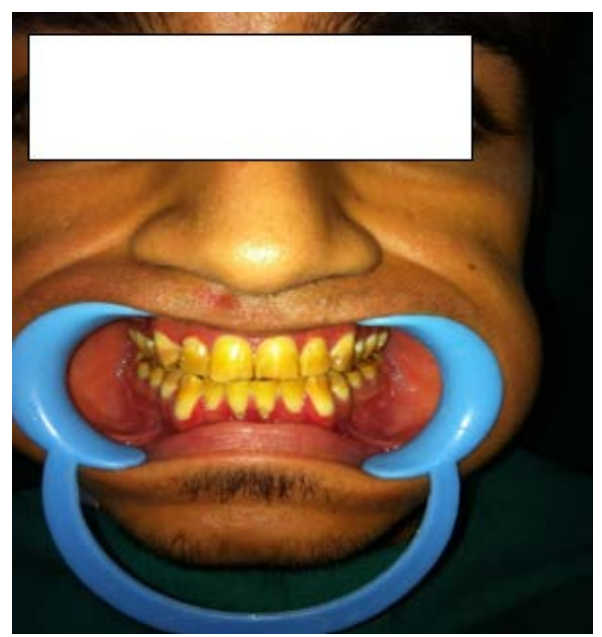

Figure 3. Intraoral brown-pigmented view of his teeth

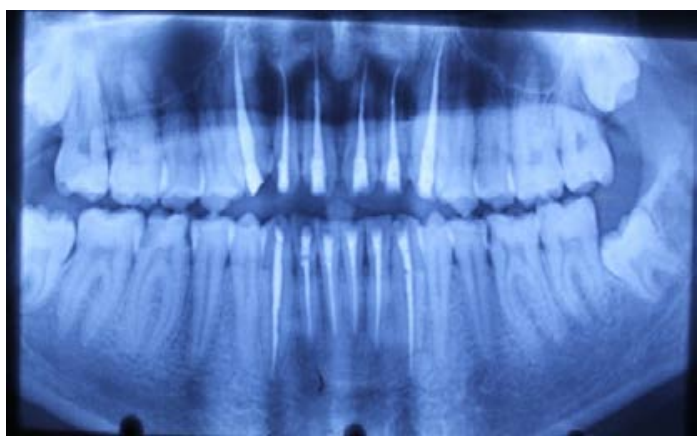

Figure 4. Orthopantograph showing endodontic treatment of anterior teeth

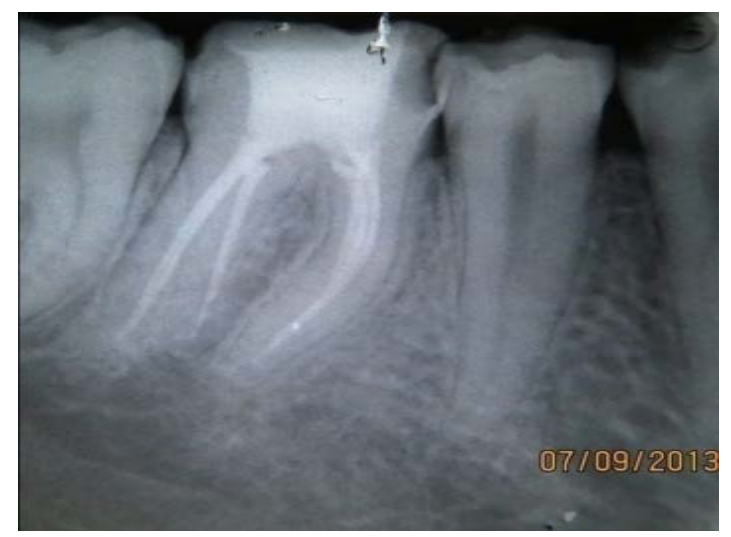

Figure 5. Endodontic treatment done for right lower molar, the proximal caries was present in this tooth

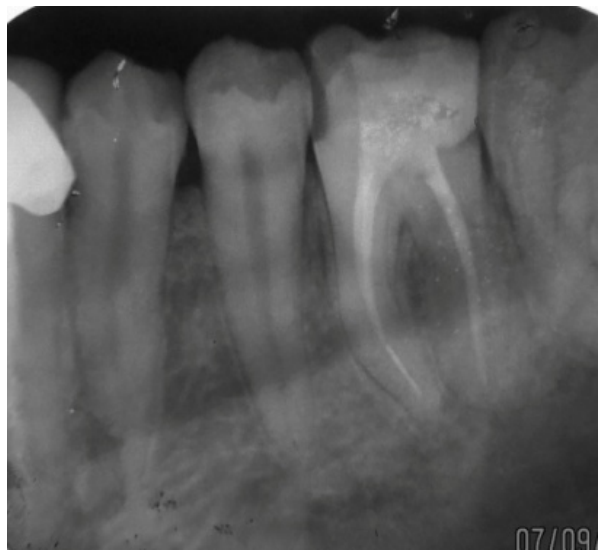

Figure 6. Intraoral periapical radiograph showing endodontic treatment of left lower molar 


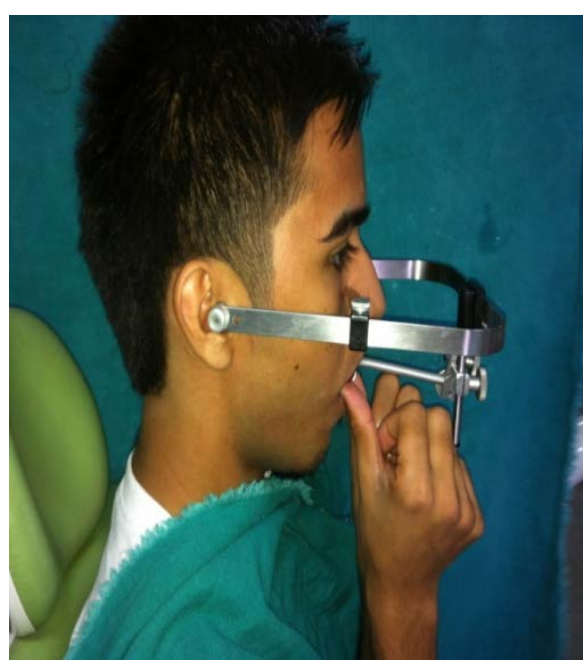

Figure 7. Face bow record

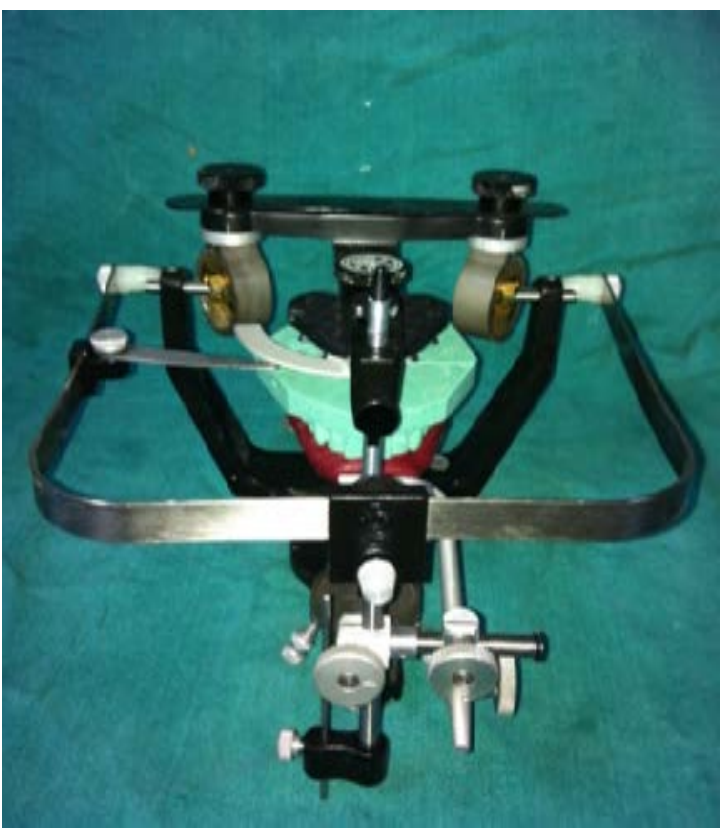

Figure 8. Face bow transfer

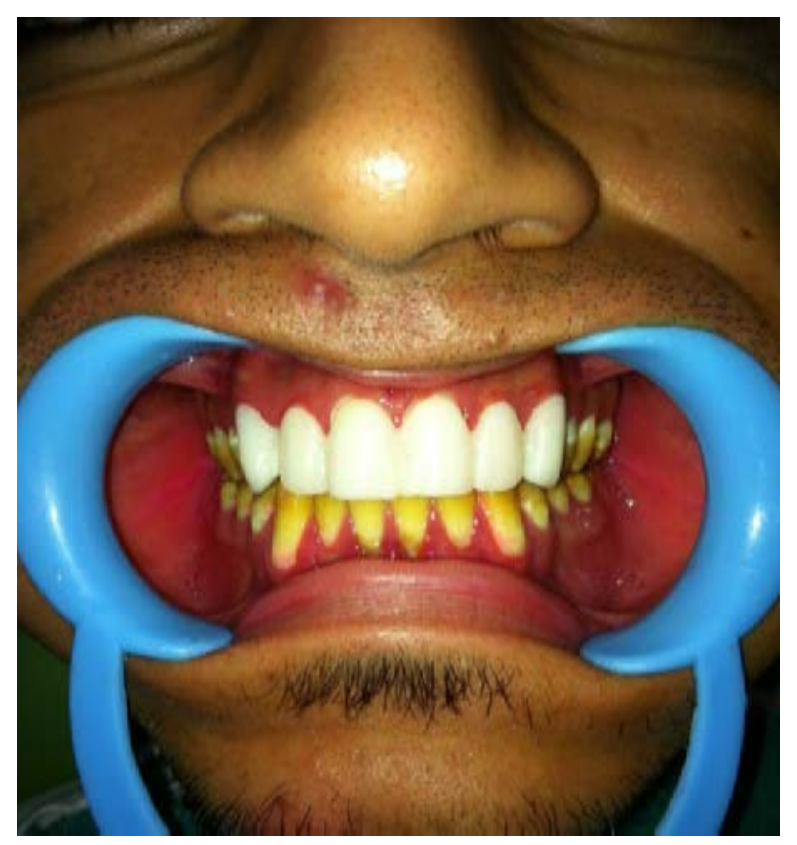

Figure 9. Provisional restoration in maxillary anteriors

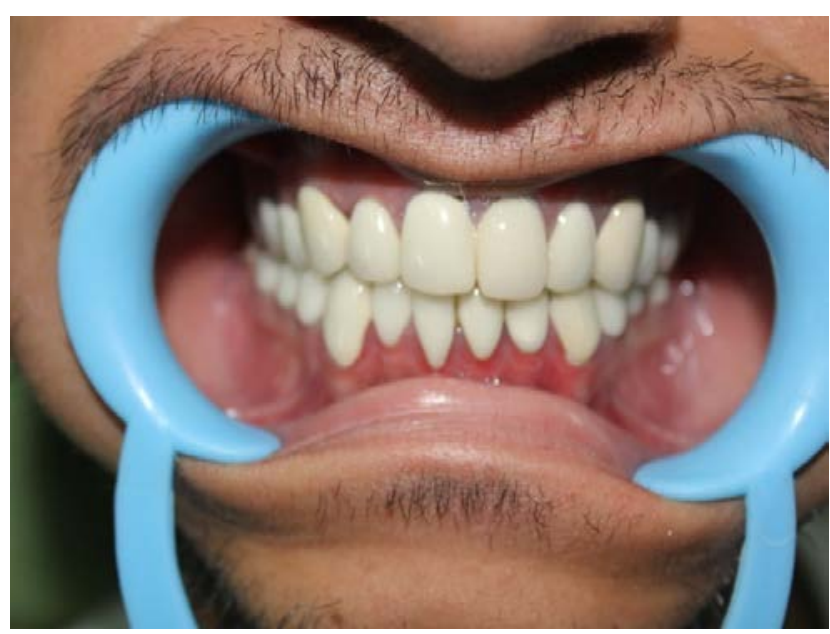

Figure 10. Final restoration in occlusion

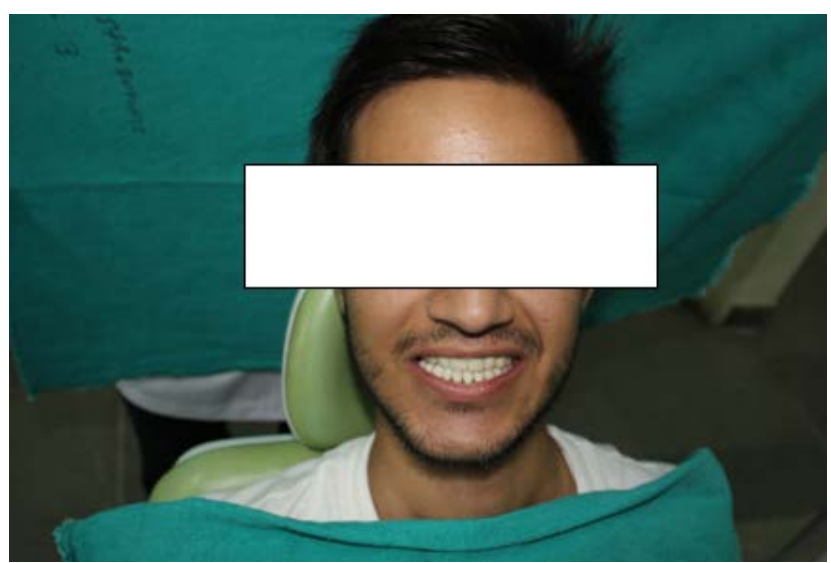

Figure 11. Smile view of the patient

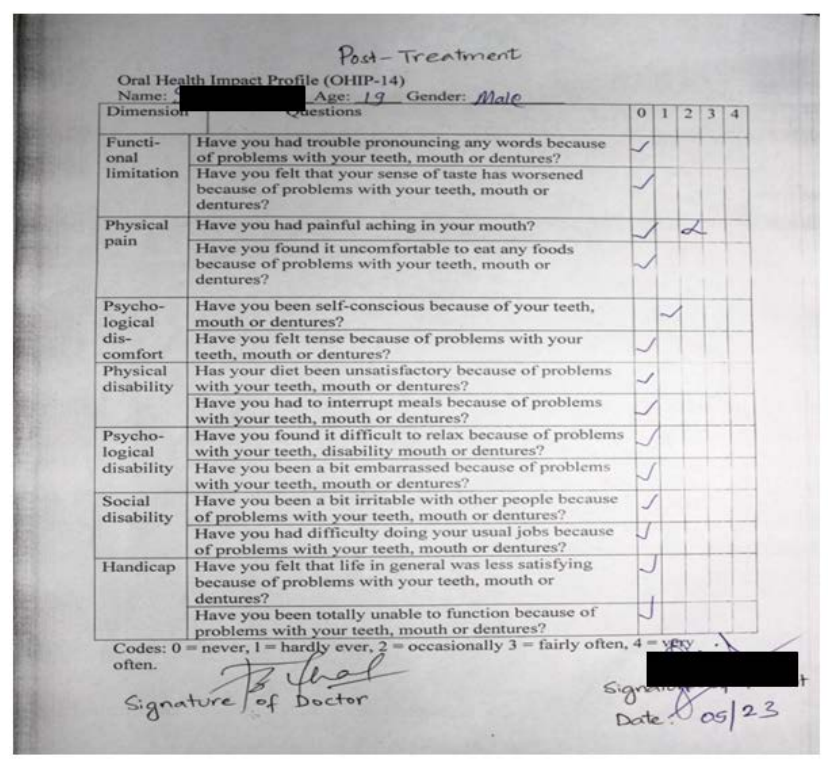

Figure 12. Post-treatment OHIP-14 form filled by the patient

\section{Conclusion}

The restoration of esthetics and functions for these kind of patients with meticulously done metal-ceramic crown results in better oral health impact profile. 


\section{Reference}

[1] A textbook of oral pathology. $4^{\text {th }}$ ed. Shafer WG, Hine MK, Levy BM. Gopsons Papers Limited: India, 1997.

[2] Berkovitz BKB, Holland GR, Moxham BJ. Oral anatomy, histology and embryology. $4^{\text {th }}$ ed. Elsevier: China; 2009.

[3] Witkop CJ. Amelogenesis imperfecta, dentinogenesis imperfecta and dentin dysplasia revisited: problems in classification. J Oral Pathol. 1989; 17 (9): 547-53.

[4] Backman B, Holm AK. Amelogenesis imperfecta: prevalence and incidence in a Northern Swedish country. Community Dent Oral Epidemiol. 1986; 14 (1): 43-7.
[5] Gupta SK, Saxena P, Jain S, Jain D. Prevalence and distribution of selected dental anomalies in an Indian population. J Oral Sci. 2011 Jun; 53 (2): 231-8

[6] Hobo S. Twin table technique for occlusal rehabilitation: part Imechanism of anterior guidance. J Prosthet Dent. 19991; 66 (3): 299-303.

[7] Hobo S. Twin table technique for occlusal rehabilitation: part II clinical procedure. J Prosthet Dent. 1991; 66 (4): 471-7.

[8] Mann AW, Pankey LD. The Pankey Mann philosophy of occlusal rehabilitation. Dent Clin North Am. 1963; 7: 621-38.

[9] Slade GD, Spencer AJ. Development and evaluation of the oral health impact profile. Community Dent Health. 1994; 11: 3-11.

[10] Slade GD. Measuring oral health and quality of life. Chapel Hill University of North Carolina, Dental Ecology, 1997. 